\title{
Relationship between weight of rescuer and quality of chest compression during cardiopulmonary resuscitation
}

\author{
Tomoyuki Hasegawa ${ }^{1,2^{*}}$, Rie Daikoku', Shin Saito ${ }^{2}$ and Yayoi Saito ${ }^{1}$
}

\begin{abstract}
Background: According to the guidelines for cardiopulmonary resuscitation (CPR), the rotation time for chest compression should be about $2 \mathrm{~min}$. The quality of chest compressions is related to the physical fitness of the rescuer, but this was not considered when determining rotation time. The present study aimed to clarify associations between body weight and the quality of chest compression and physical fatigue during CPR performed by 18 registered nurses (10 male and 8 female) assigned to light and heavy groups according to the average weight for each sex in Japan.

Methods: Five-minute chest compressions were then performed on a manikin that was placed on the floor. Measurement parameters were compression depth, heart rate, oxygen uptake, integrated electromyography signals, and rating of perceived exertion. Compression depth was evaluated according to the ratio (\%) of adequate compressions (at least $5 \mathrm{~cm}$ deep).

Results: The ratio of adequate compressions decreased significantly over time in the light group. Values for heart rate, oxygen uptake, muscle activity defined as integrated electromyography signals, and rating of perceived exertion were significantly higher for the light group than for the heavy group.

Conclusion: Chest compression caused increased fatigue among the light group, which consequently resulted in a gradual fall in the quality of chest compression. These results suggested that individuals with a lower body weight should rotate at 1-min intervals to maintain high quality CPR and thus improve the survival rates and neurological outcomes of victims of cardiac arrest.
\end{abstract}

Keywords: Cardiopulmonary resuscitation, Chest compression, Rescuer's physique, Fatigue, Rotation time, Weight, Nurse

\section{Background}

Cardiopulmonary resuscitation (CPR) comprises a series of lifesaving actions that has traditionally integrated chest compression and breathing to optimize circulation and oxygenation to improve the likelihood of survival after cardiac arrest [1,2]. The 2010 American Heart Association (AHA) guidelines for CPR and emergency cardiovascular care (ECC) suggest that all rescuers, regardless of training, should provide chest compression to victims of cardiac arrest [1]. Providing early effective chest compression for

\footnotetext{
* Correspondence: tomoyuki.hasegawa@mcn.ac.jp

'Graduate School of Health Care Sciences, Tokyo Medical and Dental University, 1-5-45 Yushima Bunkyo-ku, Tokyo 113-8510, Japan

${ }^{2}$ Mie Prefectural College of Nursing, 1-1-1 Yumegaoka, Tsu-shi, Mie 514-0116, Japan
}

such victims improves the chances of both survival and neurologically favorable outcomes [3-6].

Uninterrupted chest compression causes physical fatigue in rescuers and decreases the number of adequately deep chest compressions $[7,8]$. Current guidelines emphasize the importance of pushing hard and fast and of minimizing interruptions during compression [1]. Therefore, following these guidelines will result in a rapid decline in the quality of chest compression. If more rescuers are available, they should rotate the application of compression every $2 \mathrm{~min}$. Although the quality of chest compressions is related to the physical fitness of the rescuer [9-11], the guidelines do not consider this when determining the rotation time. 
Most cardiac arrests in hospitals are witnessed by nurses [12], whose key role in such emergencies is to apply initial CPR. Most institutions require that nurses be trained in basic life support, since nursing competence in CPR critically affects the outcomes of cardiac arrest [13]. The total number of nurses practicing in Japan during 2011 was 1,027,337, about 95\% of whom are women [14] and about half of them are aged in their 20 s or 30s. Any individual in a hospital should be able to provide the best possible quality chest compression regardless of physical type. Therefore, rotation time rather than uniform rotation time considering the physique of rescuer should be considered to ensure the most effective chest compression. The quality of chest compression positively correlates with the height of the rescuer [10], but the relationship with the weight of the rescuer has not been proven. The Japanese physique is generally smaller than that of Europeans and Americans and although the weight of rescuers might influence the quality of chest compression, rotation time should be determined according to a smaller physique.

The present study aimed to clarify associations between the quality of chest compression and body weight as well as the physical fatigue of rescuers. We propose that the amount of rotation time required to deliver the most effective chest compression delivered by persons with a lower body weight should be decreased to deliver more effective cardiopulmonary resuscitation to ensure optimal outcomes for victims of cardiac arrest.

\section{Methods}

\section{Participants}

The present study included 18 (male, $\mathrm{n}=10$; female, $\mathrm{n}=8$ ) registered nurses employed in emergency, intensive care, or cardiovascular medicine departments who have current certifications in basic life support and have delivered inhospital CPR. None of them had musculoskeletal or functional mobility issues. All of them provided written, informed consent to participate in this study, which was approved by the Mie Prefectural College of Nursing. Information was then collected about sex, age, clinical experience, height, weight, body mass index (BMI), and exercise habits (Table 1 ). None of the nurses regularly participated in exercise.

Table 1 Characteristics of participants

\begin{tabular}{lccc}
\hline & Male & Female & Total \\
& $\mathbf{n = 1 0}$ & $\mathbf{n = 8}$ & $\mathbf{n = 1 8}$ \\
\hline Age (years) & $28.1 \pm 3.6$ & $29.1 \pm 2.3$ & $28.5 \pm 3.1$ \\
Height $(\mathrm{cm})$ & $173.8 \pm 6.3$ & $158.0 \pm 4.2$ & $166.8 \pm 9.6$ \\
Weight $(\mathrm{kg})$ & $64.5 \pm 10.6$ & $53.4 \pm 10.2$ & $59.7 \pm 11.8$ \\
BMl $\left(\mathrm{kg} / \mathrm{m}^{2}\right)$ & $21.3 \pm 3.8$ & $21.4 \pm 3.9$ & $21.3 \pm 3.8$ \\
\hline
\end{tabular}

Values are means \pm standard deviation.

\section{Protocol}

This study protocol comprised rest and chest compression in that order for $5 \mathrm{~min}$ each (Figure 1). The nurses practiced chest compression until they could consistently apply at least $5 \mathrm{~cm}$ of compression. During the 5min rest period, they remained seated with their eyes closed while wearing a face-mask and electromyography (EMG) and electrocardiography (ECG) electrodes. Fiveminute chest compressions were then performed on a Resusci Anne Skill Reporter (Leardal Medical Corporation, Stavanger, Norway) that was placed on the floor without audiovisual feedback (Figure 2). They maintained a compression rate of $100 / \mathrm{min}$ by following a metronome [15]. At the end of the chest compressions, they recovered in a comfortable posture.

\section{Data collection}

Compression depth (CD) was captured as each participant performed CPR on a Resusci Anne Skill Reporter. Heart rate was measured at rest and during chest compression using a Life Scope 8 (NIHON KOHDEN Co., Tokyo, Japan). Oxygen uptake (VO2) during chest compression was continuously measured using a VO2000 (S\&ME Co., Raleigh, NC, USA). Surface electromyography (sEMG) captured data from the biceps brachii, triceps brachii, trapezius, erector spinae, external oblique muscle, abdominal rectus muscle, rectus femoris, and biceps femoris during chest compression [16,17]. Two electrodes were attached to the belly of each muscle at an inter-electrode distance of $2.5 \mathrm{~cm}$. The skin was abraded and cleaned with alcohol before attaching electrodes to minimize impedance. Analog HR and EMG signals were sampled using an AD16-16(PCI) E A/D converter (CONTEC CO. Ltd., Osaka, Japan) at $3 \mathrm{KHz}$, and stored in a personal computer using a G1 system Analog Recorder Pro Ver. 1.60 (G1 system, Aichi, Japan). The rating of perceived exertion (RPE) was rated using Borg's 15 point scale (range, 6 to 20).

\section{Evaluation}

The nurses were assigned to light ( 5 male and 4 female) and heavy (male, $\mathrm{n}=5$; female, $\mathrm{n}=4$ ) groups according to the average weight for each sex in Japan [18]. Compression depth (CD) was evaluated according to the ratio (\%) of adequate compressions (at least $5 \mathrm{~cm}$ deep) per 30 s [19]. Heart rate and VO2 were evaluated during both chest compression based on changes from the initial baseline value after rest. The sEMG signals were full-wave rectified and integrated over a period of $30 \mathrm{~s}$ during chest compressions to determine muscle activity (iEMG) [17]. Physical fatigue was determined by matching against an RPE scale during chest compression every minute. 


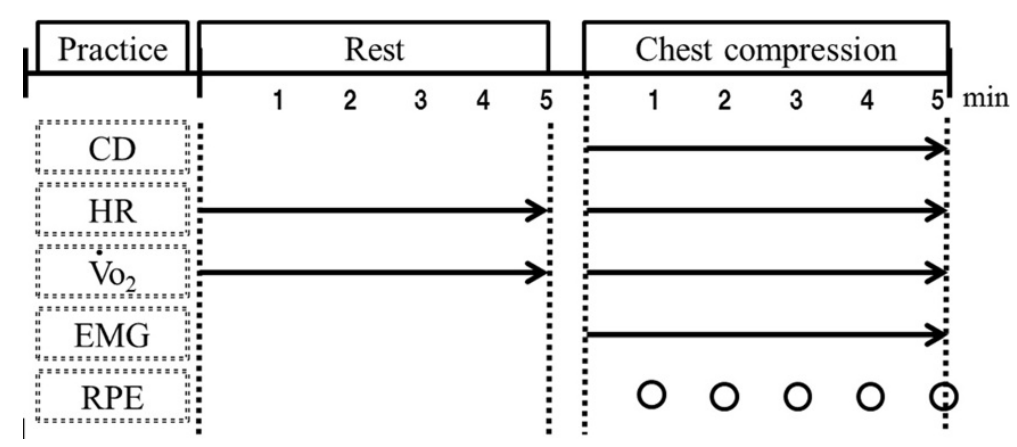

Figure 1 Experimental protocol. Compression depth (CD), heart rate (HR), oxygen uptake (VO2), electromyography (EMG), and rating of perceived exertion (RPE) were measured.

\section{Statistical analysis}

The iEMG values were compared between the heavy and light groups using independent-sample $t$ tests and a one-way analysis of variance. The ratio (\%) of adequate compressions as well as values for HR, VO2 and RPE were analyzed using the Mann-Whitney $U$ test, the Friedman test, and the Wilcoxon sign rank test. All data were statistically analyzed using SPSS/PASW Statistics Ver. 18.0 (IBM, Armonk, NY, USA). The significance level was set at 0.05 .

\section{Ethics}

The Ethics Review board at Mie Prefectural College of Nursing approved the study (Approval No. 120203). The experimental protocols and procedures were explained to all those who responded to public advertisements about the study and then all voluntarily provided written, informed consent to participate. High priority was given to the safety of the participants and appropriate measures were taken to process the withdrawal of any participant from the study at any time.

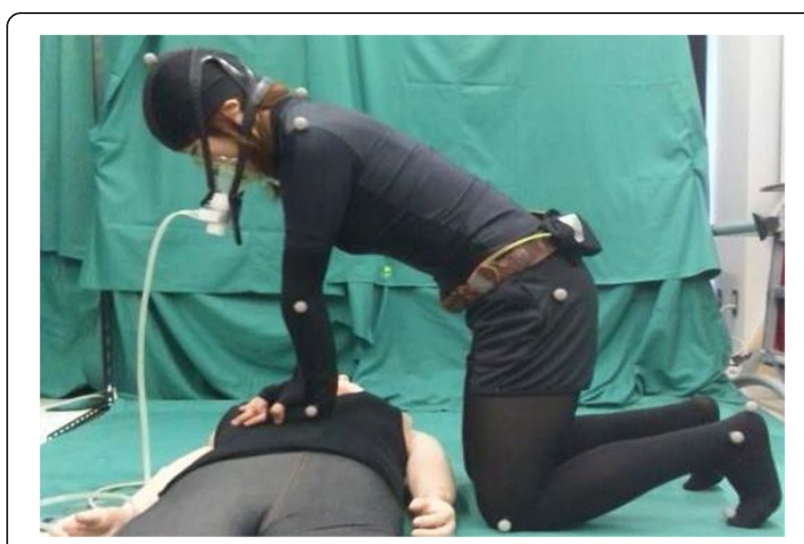

Figure 2 Posture for chest compression.

\section{Results}

Participants

The average weight and BMI of the light and heavy groups significantly differed $(50.6 \pm 6.5 v s .68 .0 \pm 7.5 \mathrm{~kg}$, $18.2 \pm 1.6$ vs. $24.4 \pm 2.8 \mathrm{~kg} / \mathrm{m}^{2}, P<0.001$; Table 2), whereas age, clinical experience, height, $\mathrm{HR}$, and $\mathrm{VO} 2$ at rest did not. All participants were capable of applying chest compression for $5 \mathrm{~min}$.

\section{Compression depth}

The median ratios (\%) of adequate compression for each 30-s interval by the light and heavy groups ranged from 77.4 to 0.0 and 98.1 to 77.3 , respectively (Figure 3 ). The ratio of adequate compression applied by the heavy group did not significantly decline, but significantly decreased over time from 150, 180, 210, 240, 270, and $300 \mathrm{~s}(P=0.028)$ in the light group. Values significantly differed at $90,150,180,210,240,270$, and $300 \mathrm{~s}(P=$ $0.027,0.049,0.049,0.035,0.035,0.022,0.035$, respectively) between the groups.

\section{Heart rate}

The median value of HR (bpm) for chest compression compared with that at rest for each 30-s interval ranged from 134.3 to 109.9 and 118.2 to 97.2 in the light and heavy groups, respectively (Figure 4). These values significantly differed between the groups at $60,90,120$, $150,180,210,240$, and $270 \mathrm{~s}(P=0.043,0.020,0.008$, $0.008,0.013,0.029,0.029,0.043$, respectively) during chest compression.

\section{Oxygen uptake}

The median value of $\mathrm{VO} 2(\mathrm{~mL} / \mathrm{kg} / \mathrm{min})$ for chest compression compared with that at rest for each 30 -s interval ranged from 16.3 to 8.9 and 12.9 to 7.6 in the light and heavy groups, respectively (Figure 5 ). Values significantly differed between the groups during chest compression at 90,120,150,180,210, 240, 270, and $300 \mathrm{~s}$ 
Table 2 Characteristics of groups

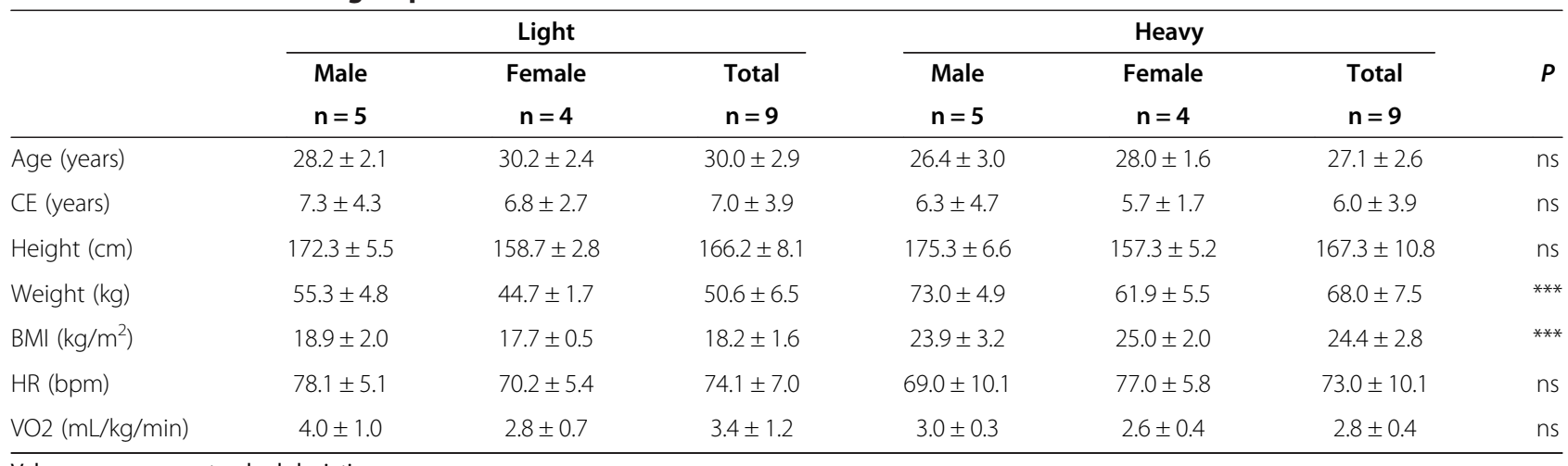

Values are means \pm standard deviation.

${ }^{* * *} P<0.001$ (unpaired $t$ test) between totals of light and heavy groups.

$\mathrm{CE}$, clinical experience; ns, no significance.

$(P=0.005,0.020,0.014,0.007,0.007,0.014,0.005,0.020$, respectively).

\section{Muscle activity}

Mean iEMG (mV) values for the trapezius, erector spinae, external oblique muscle, abdominal rectus muscle, and rectus femoris significantly differed between the light and heavy groups (Table 3), whereas those for the biceps brachii, triceps brachii, and biceps femoris did not significantly differ between the groups. Muscle activity did not significantly change over time against the value after $30 \mathrm{~s}$ in either group.

\section{Rating of perceived exertion}

The median values for rating of perceived exertion (RPE) for each 1-min interval ranged from 17.5 to 12.0 and 15.0 to 11.0 in the light and heavy groups, respectively (Figure 6). The RPE significantly increased over time against the value for the first minute for 2,3 , 4, and
5 in both groups. Values significantly differed between the groups at $2,3,4$, and $5 \min (P=0.014,0.010,0.021$, 0.018 , respectively).

\section{Discussion}

The quality of chest compression delivered during CPR using only the hands decreases over time $[1-3,8,16,19,20]$. The present study confirmed that the quality of chest compression by the light group changed as described $[7,8]$ and this group became more fatigued thereafter.

Chest compression requires power to be applied from a point that is vertically above the sternum to a depth of $5 \mathrm{~cm}$. The amount of power required to depress a sternum by $5 \mathrm{~cm}$ is about $500 \mathrm{~N}$ [21-23]. Chest compression force during CPR is generated using gravity and hip flexion torque [16]. Those applying chest compression develop force by accelerating the upper body downwards using gravity [16] and use hip extension torque to hold the trunk up at decompression, which resists the inertial

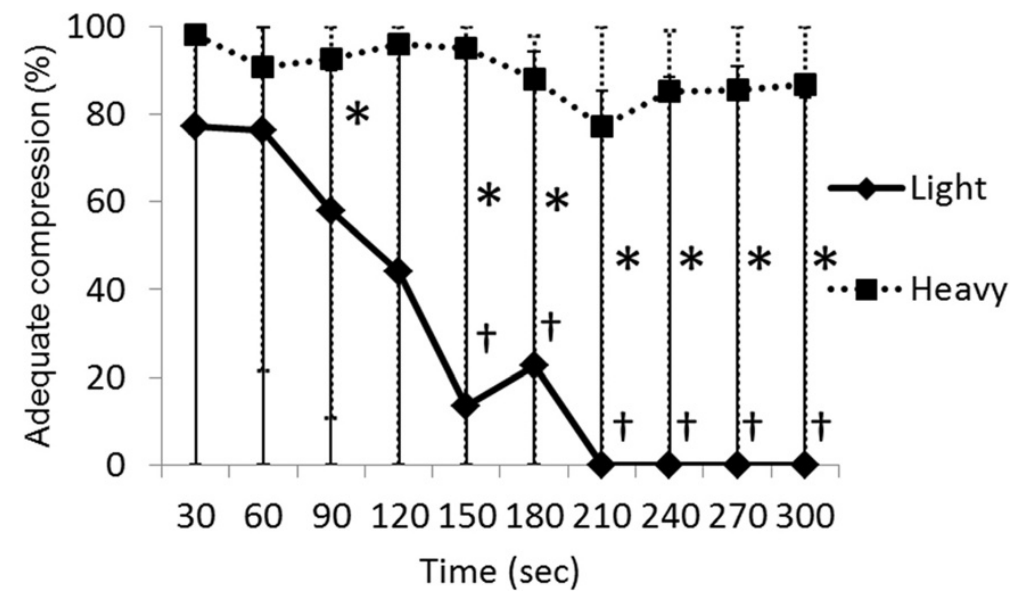

Figure 3 Comparison of ratios of adequate compression between light and heavy groups. Values are shown as medians and max-min. Significant differences between light and heavy groups ( ${ }^{*} P<0.05$; Mann-Whitney $U$ test) and between 30 s and subsequent times in light group $\left({ }^{\dagger} P<0.05\right.$; Wilcoxon sign rank test). 


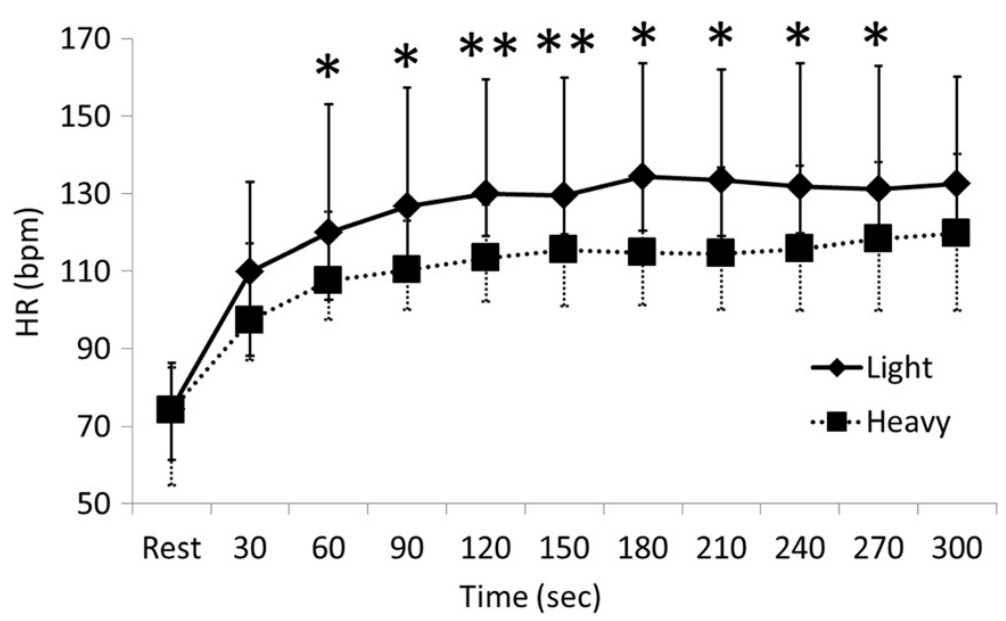

Figure 4 Comparison of heart rates between light and heavy groups. Values are shown as medians and max-min. Significant difference between light and heavy groups ( ${ }^{*} P<0.05$ and ${ }^{* *} P<0.01$; Mann-Whitney $U$ test). HR, heart rate.

force of gravity [16]. The moment acting at the lumbar spine represents the loads generated by muscles during chest compression [24].

The light group produced the required force for pressure by utilizing the trapezius, abdominal rectus, external oblique, and rectus femoris muscles. At the moment of decompression, force enters the erector spinae, which is an antagonist of compression, and this might explain the increased physical fatigue in the light group compared with the heavy group.

For this reason, a lighter rescuer quickly becomes subjectively and objectively fatigued in during hands-only $\mathrm{CPR}$, and the quality of chest compression rapidly deteriorates. Furthermore, one of our participants was unable to compress the sternum to a depth of $5 \mathrm{~cm}$ from the start of the experiment. Either debriefing or feedback alone improves CPR quality, but the combination of both improves performance more effectively [25]. An audiovisual cardiopulmonary resuscitation feedback device has significantly improved the quality of chest compression provided by experienced hospital nurses in a simulated setting [26]. We did not use an audiovisual feedback device in the present study and assumed that some participants in the light group had forgotten what they had learned while practicing chest compression before starting the experiments.

On the other hand, the heavy group maintained the ratio of adequate compression at $>70 \%$ for $5 \mathrm{~min}$. This group was able to generate sufficient compression force without having to involve the muscular power of the trunk or thigh, unlike the light group. Chest compression by the heavy group utilized the rescuer's weight as a compression force. Good physical fitness and the height of the rescuer correlate positively and independently of

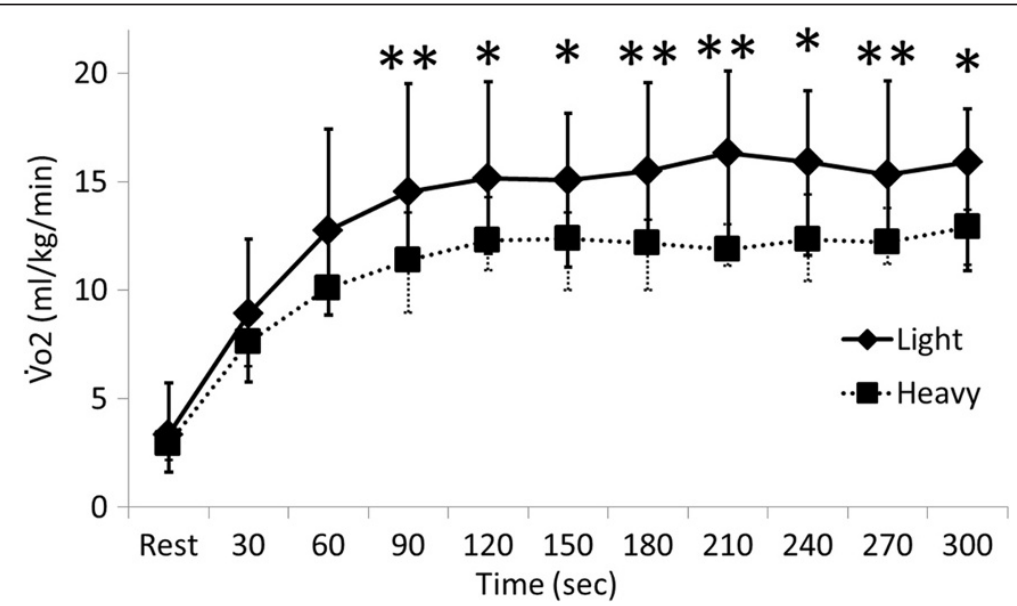

Figure 5 Comparison of oxygen uptake between light and heavy groups. Values are shown as medians and max-min. Significant difference between light and heavy groups ( ${ }^{*} P<0.05$ and ${ }^{* *} P<0.01$; Mann-Whitney $U$ test). VO2, oxygen uptake. 
Table 3 Comparison of iEMG values between light and heavy groups

\begin{tabular}{|c|c|c|c|c|c|c|c|c|c|c|}
\hline & 30 & 60 & 90 & 120 & 150 & 180 & 210 & 240 & 270 & $300(s)$ \\
\hline \multicolumn{11}{|c|}{ Biceps brachii (mV) } \\
\hline Light & $27.3 \pm 12.8$ & $26.5 \pm 12.5$ & $24.5 \pm 10.1$ & $25.1 \pm 11.0$ & $23.5 \pm 9.5$ & $24.3 \pm 9.6$ & $22.2 \pm 8.7$ & $23.9 \pm 10.1$ & $22.2 \pm 9.7$ & $23.2 \pm 10.6$ \\
\hline Heavy & $28.5 \pm 9.5$ & $26.2 \pm 6.9$ & $24.0 \pm 6.2$ & $23.2 \pm 6.9$ & $22.7 \pm 6.9$ & $22.7 \pm 7.4$ & $22.2 \pm 8.0$ & $22.9 \pm 8.7$ & $22.9 \pm 9.1$ & $22.3 \pm 9.2$ \\
\hline \multicolumn{11}{|c|}{ Triceps brachii (mV) } \\
\hline Light & $69.5 \pm 25.6$ & $67.4 \pm 22.5$ & $65.7 \pm 20.5$ & $65.6 \pm 18.2$ & $67.3 \pm 18.8$ & $70.3 \pm 18.8$ & $68.7 \pm 18.2$ & $68.6 \pm 17.0$ & $68.4 \pm 15.4$ & $70.4 \pm 16.8$ \\
\hline Heavy & $50.1 \pm 9.0$ & $51.1 \pm 10.3$ & $50.4 \pm 11.3$ & $52.0 \pm 12.7$ & $51.2 \pm 13.5$ & $52.5 \pm 16.0$ & $53.0 \pm 16.0$ & $55.2 \pm 18.9$ & $53.7 \pm 17.0$ & $55.8 \pm 16.5$ \\
\hline \multicolumn{11}{|c|}{ Trapezius (mV) } \\
\hline Light & $26.8 \pm 14.8$ & $25.1 \pm 14.5$ & $21.9 \pm 11.2$ & $21.0 \pm 10.2$ & $20.8 \pm 9.6$ & $21.9 \pm 9.4$ & $21.1 \pm 8.0$ & $22.3 \pm 9.2$ & $21.8 \pm 7.2$ & $25.5 \pm 10.1$ \\
\hline Heavy & $14.3 \pm 4.8^{*}$ & $13.7 \pm 4.7$ & $13.3 \pm 4.9$ & $13.5 \pm 5.4$ & $14.2 \pm 9.0$ & $13.6 \pm 7.9$ & $12.7 \pm 5.2^{*}$ & $12.1 \pm 3.9^{*}$ & $12.3 \pm 5.4^{* *}$ & $13.3 \pm 5.8^{* *}$ \\
\hline \multicolumn{11}{|c|}{ Erector spinae $(\mathrm{mV})$} \\
\hline Light & $19.0 \pm 5.4$ & $19.8 \pm 6.3$ & $19.4 \pm 5.7$ & $19.1 \pm 5.0$ & $18.7 \pm 5.1$ & $19.5 \pm 5.4$ & $19.1 \pm 5.2$ & $20.0 \pm 4.8$ & $20.0 \pm 4.7$ & $20.5 \pm 4.4$ \\
\hline Heavy & $12.2 \pm 4.9^{*}$ & $12.8 \pm 6.0^{*}$ & $13.0 \pm 5.4^{*}$ & $12.7 \pm 4.8^{*}$ & $12.9 \pm 4.5^{*}$ & $12.9 \pm 3.9^{*}$ & $13.1 \pm 4.3^{*}$ & $13.2 \pm 4.3^{* *}$ & $13.2 \pm 4.8^{* *}$ & $13.4 \pm 5.1^{* *}$ \\
\hline \multicolumn{11}{|c|}{ External oblique (mV) } \\
\hline Light & $22.0 \pm 9.4$ & $25.9 \pm 14.2$ & $22.6 \pm 12.0$ & $22.4 \pm 11.8$ & $19.6 \pm 8.8$ & $21.6 \pm 9.6$ & $20.6 \pm 8.8$ & $22.2 \pm 10.2$ & $21.0 \pm 9.2$ & $22.0 \pm 10.5$ \\
\hline Heavy & $11.3 \pm 6.3^{*}$ & $10.9 \pm 5.4^{*}$ & $10.3 \pm 4.9^{*}$ & $9.6 \pm 4.1^{*}$ & $9.5 \pm 4.6^{*}$ & $9.5 \pm 4.4^{* *}$ & $9.3 \pm 4.5^{* *}$ & $9.1 \pm 4.1^{* *}$ & $8.6 \pm 3.9^{* *}$ & $8.3 \pm 3.2^{* *}$ \\
\hline \multicolumn{11}{|c|}{ Abdominal rectus (mV) } \\
\hline Light & $18.4 \pm 6.7$ & $19.3 \pm 10.3$ & $18.2 \pm 9.6$ & $18.4 \pm 11.2$ & $17.3 \pm 10.0$ & $18.1 \pm 9.5$ & $17.3 \pm 9.5$ & $17.9 \pm 10.7$ & $17.6 \pm 9.3$ & $17.7 \pm 9.5$ \\
\hline Heavy & $8.7 \pm 3.5^{* *}$ & $8.4 \pm 3.3^{*}$ & $8.0 \pm 2.7^{*}$ & $7.7 \pm 2.7^{*}$ & $7.0 \pm 2.4^{*}$ & $7.2 \pm 2.4^{* *}$ & $7.0 \pm 2.5^{* *}$ & $7.2 \pm 2.8^{*}$ & $7.0 \pm 2.6^{* *}$ & $7.3 \pm 2.9^{*}$ \\
\hline \multicolumn{11}{|c|}{ Rectus femoris (mV) } \\
\hline Light & $7.2 \pm 2.3$ & $8.6 \pm 4.1$ & $10.6 \pm 5.6$ & $11.1 \pm 6.7$ & $10.5 \pm 6.8$ & $11.2 \pm 7.2$ & $9.7 \pm 5.9$ & $10.2 \pm 7.1$ & $10.7 \pm 6.5$ & $11.1 \pm 7.9$ \\
\hline Heavy & $4.3 \pm 0.8^{* *}$ & $4.1 \pm 0.7^{*}$ & $3.7 \pm 0.7^{* *}$ & $3.7 \pm 0.7^{*}$ & $3.5 \pm 0.7^{*}$ & $3.7 \pm 1.1^{*}$ & $3.4 \pm 0.9^{*}$ & $3.7 \pm 1.1^{*}$ & $3.9 \pm 1.7^{*}$ & $3.5 \pm 0.8^{*}$ \\
\hline \multicolumn{11}{|c|}{ Biceps femoris (mV) } \\
\hline Light & $32.3 \pm 24.8$ & $28.5 \pm 18.2$ & $24.0 \pm 17.1$ & $22.1 \pm 15.3$ & $23.3 \pm 15.4$ & $23.8 \pm 15.5$ & $24.3 \pm 16.7$ & $26.4 \pm 16.7$ & $26.4 \pm 17.1$ & $26.8 \pm 15.7$ \\
\hline Heavy & $14.1 \pm 6.6$ & $15.2 \pm 7.1$ & $15.1 \pm 5.6$ & $15.1 \pm 5.5$ & $15.3 \pm 5.5$ & $14.7 \pm 5.4$ & $14.5 \pm 4.8$ & $14.5 \pm 4.9$ & $14.6 \pm 4.5$ & $14.5 \pm 4.2$ \\
\hline
\end{tabular}

Values are means \pm standard deviation. Significant difference between light and heavy groups. $\left({ }^{*} P<0.05,{ }^{* *} P<0.01\right.$; unpaired $t$ test); $n=9$ for all groups.

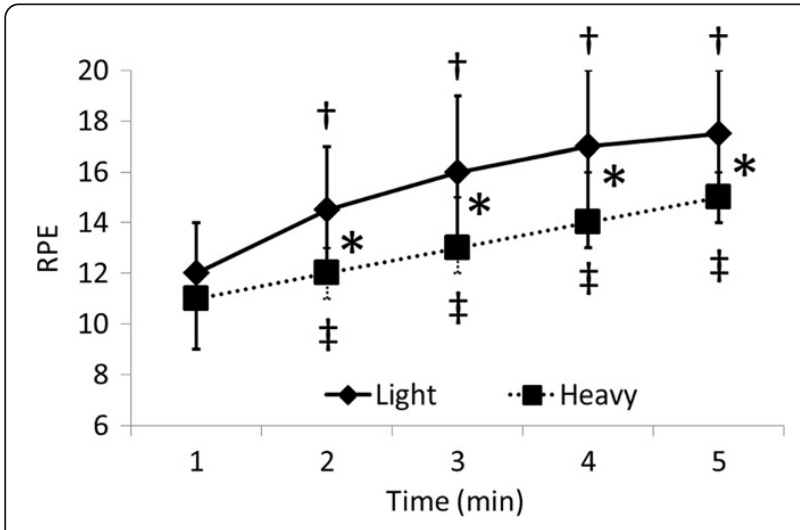

Figure 6 Comparison of RPE between light and heavy groups. Values are shown as medians and max-min. Significant difference between light and heavy groups $\left({ }^{*} P<0.05\right.$; Mann-Whitney $U$ test) and between 1 min and subsequent times in light $\left(^{\dagger} P<0.05\right.$; Wilcoxon sign rank test) and heavy $\left({ }^{\ddagger} P<0.05\right.$; Wilcoxon sign rank test) groups. RPE, rating of perceived exertion. sex with the quality of chest compression [10,11]. Our data are in agreement with these findings. The heavy group could use their body weight to provide chest compression without becoming quickly fatigued, indicating that the weight of the rescuer is an important element of effective chest compression.

Effective chest compressions are essential for providing blood flow during CPR. For this reason all patients in cardiac arrest should receive chest compression [1]. To provide effective chest compression, push hard and push fast. It is reasonable for laypersons and healthcare providers to compress the adult chest at a rate of at least 100 compressions per minute with a compression depth at least $5 \mathrm{~cm}$ [1]. Chest compression in hospital is often insufficient even when applied by medical staff [27]. According to the guidelines, the rotation time for chest compression should be about $2 \mathrm{~min}$, regardless of physical type. However, the physical features of rescuers must be considered to provide and maintain high quality CPR. With respect to the rotation time for chest compression in particular, an index could be constructed 
using the body weight of rescuers as one factor. This study suggests that rescuers of low weight should rotate every minute to maintain effective CPR.

\section{Conclusions}

The light group became fatigued while delivering chest compression, which gradually decreased the quality of the compression. On the other hand, the heavy group could apply effective chest compression for $5 \mathrm{~min}$. The weight of the rescuer is an important factor in the quality of chest compression. The ratio of adequate compression significantly differed after $1 \mathrm{~min}$, suggesting that individuals of light weight should rotate at intervals of $1 \mathrm{~min}$

\section{Abbreviations \\ AHA: American Heart Association; BMl: Body mass index; CD: Compression depth; CE: Clinical experience; CPR: Cardiopulmonary resuscitation; ECC: Emergency cardiovascular care; ECG: Electrocardiogram; \\ EMG: Electromyogram; HR: Heart rate; iECG: Integrated electrocardiograph; RPE: Rating of perceived exertion; sECG: Surface electrocardiography; VO2: oxygen uptake.}

\section{Competing interests}

The authors declare that they have no competing interests.

\section{Authors' contributions}

TH performed the experiments, analyzed the data and wrote the manuscript. DR, SS, and YS coordinated the study and supervised the collection and analysis of data. All authors have read and approved the final manuscript.

\section{Acknowledgments}

This study was supported by a Grant-in-Aid for Young Scientists (B) (24792405) from the Japan Society for the Promotion of Science. We thank the study participants and Prof. Myles O'Brien of Mie Prefectural College of Nursing for providing English language advice.

Received: 16 January 2014 Accepted: 13 June 2014

Published: 24 June 2014

\section{References}

1. Berg RA, Hemphill R, Abella BS, Aufderheide TP, Cave DM, Hazinski MF, Lerner EB, Rea TD, Sayre MR, Swor RA: Part 5: adult basic life support: 2010 American heart association guidelines for cardiopulmonary resuscitation and emergency cardiovascular care. Circulation 2010, 122:S685S705.

2. Koster RW, Baubin MA, Bossaert LL, Caballero A, Cassan P, Castrén M, Granja C, Handley AJ, Monsieurs KG, Perkins GD, Raffay V, Sandroni C: European resuscitation council guidelines for resuscitation 2010 section 2. Adult basic life support and use of automated external defibrillators. Resuscitation 2010, 81:12771292.

3. Heidenreich JW, Bonner A, Sanders AB: Rescuer fatigue in the elderly: standard vs. hands-only CPR. J Emerg Med 2012, 42:8889.

4. Holmberg M, Holmberg S, Herlitz J: Effect of bystander cardiopulmonary resuscitation in out-of- hospital cardiac arrest patients in Sweden. Resuscitation 2000, 47:5970

5. SOS-KANTO study group: Cardiopulmonary resuscitation by bystanders with chest compression only (SOS-KANTO): an observational study. Lancet 2007, 369:920926.

6. Nagao K: Chest compression-only cardiocerebral resuscitation. Curr Opin Crit Care 2009, 15:189197.

7. Gutwirth H, Williams B, Boyle M: Rescuer fatigue in cardiopulmonary resuscitation: a review of the literature. JEPHC 2009, 7(4):19.

8. Bjørshol CA, Sunde K, Myklebust H, Assmus J, Søreide E: Decay in chest compression quality due to fatigue is rare during prolonged advanced life support in a manikin model. Scand J Trauma Resusc Emerg Med 2011, 19:46.
9. Ock SM, Kim YM, Chung J, Kim SH: Influence of physical fitness on the performance of 5-minute continuous chest compression. Eur J Emerg Med 2011, 18:251256.

10. Russo SG, Peter N, Sylvia R, Arnd T, André N, Michael Q, Eich CB: Impact of physical fitness and biometric data on the quality of external chest compression: a randomised, crossover trial. BMC Emerg Med 2011, 11:20.

11. Hansen D, Vranckx P, Broekmans T, Eijnde BO, Beckers W, Vandekerckhove P, Broos $P$, Dendale $P$ : Physical fitness affects the quality of single operator cardiocerebral resuscitation in healthcare professionals. Eur J Emerg Med 2012, 19:2834.

12. Heng KWJ, Fong MK, Wee C, Anantharaman V: The role of nurses in the resuscitation of in-hospital cardiac arrests. Singapore Med J 2011, 52:611615

13. Mokhtari J, Nori M, Saghafinia MH, Kalantar Motamedi SM, Hosseini K: CPR training for nurses: how often is it necessary? Iran Red Crescent Med J 2012, 14:104107.

14. Japanese Nursing Association Publishing Company: Statistical Data on Nursing Service in Japan 2012. Tokyo: Japanese Nursing Association Publishing Company; 2013.

15. Park Sang O, Hong Chong K, Shin Dong H, Lee Jun H, Hwang Seong Y: Efficacy of metronome sound guidance via a phone speaker during dispatcher-assisted compression-only cardiopulmonary resuscitation by an untrained layperson: a randomised heavyled simulation study using a manikin. Emerg Med J 2013, 30:657661.

16. Trowbridge C, Parekh JN, Ricard MD, Potts J, Patrickson WC, Cason CL: A randomized cross-over study of the quality of cardiopulmonary resuscitation among females performing 30:2 and hands-only cardiopulmonary resuscitation. BMC Nurs 2009, 8:13p.

17. Yasuda Y, Kato Y, Sugimoto K, Tanaka S, Tsunoda N, Kumagawa D, Toyokuni Y, Kubota K, Inaba H: Muscles used for chest compression under static and transportation conditions. Prehosp Emerg Care 2013, 17:162169.

18. Research Institute of Human Engineering for Quality Life: Japanese body size data book 2004-2006. Osaka: Research Institute of Human Engineering for Quality Life; 2011.

19. McDonald Catherine H, Heggie J, Jones Christopher M, Thorne Christopher J, Hulme J: Rescuer fatigue under the 2010 ERC guidelines, and its effect on cardiopulmonary resuscitation (CPR) performance. Emerg Med J 2013, 30:623627.

20. Foo NP, Chang JH, Lin HJ, Guo HR: Rescuer fatigue and cardiopulmonary resuscitation positions: a randomized controlled crossover trial. Resuscitation 2010, 81:579584.

21. Tomlinson AE, Nysaether J, Kramer-Johansen J, Steen PA, Dorph E: Compression force-depth relationship during out-of-hospital cardiopulmonary resuscitation. Resuscitation 2007, 72:364370.

22. Chi CH, Tsou JY, Su FC: Effects of rescuer position on the kinematics of cardiopulmonary resuscitation (CPR) and the force of delivered compressions. Resuscitation 2008, 76:6975.

23. Chi C, Tsou J, Su F: Effects of compression-to-ventilation ratio on compression force and rescuer fatigue during cardiopulmonary resuscitation. Am J Emerg Med 2010, 28:10161023.

24. Jones AYM, Lee RYW: Rescuer's position and energy consumption, spinal kinetics, and effectiveness of simulated cardiac compression. Am J Crit Care 2008, 17:417425.

25. Dine CJ, Gersh RE, Leary M, Riegel BJ, Bellini LM, Abella BS: Improving cardiopulmonary resuscitation quality and resuscitation training by combining audiovisual feedback and debriefing. Crit Care Med 2008, 36:28172822.

26. Pozner CN, Almozlino A, Elmer J, Poole S, McNamara D, Barash D: Cardiopulmonary resuscitation feedback improves the quality of chest compression provided by hospital health care professionals. Am J Emerg Med 2011, 29:618625.

27. Abella BS, Alvarado JP, Myklebust H, Edelson DP, Barry A, O'Hearn N, Vanden Hoek TL, Becker LB: Quality of cardiopulmonary resuscitation during in-hospital cardiac arrest. JAMA 2005, 293:305310.

\section{doi:10.1186/1880-6805-33-16}

Cite this article as: Hasegawa et al: Relationship between weight of rescuer and quality of chest compression during cardiopulmonary resuscitation. Journal of Physiological Anthropology 2014 33:16. 